\title{
Growing wafer-scale single-crystal hBN
}

Hexagonal boron nitride (hBN) is a promising dielectric for $2 \mathrm{D}$ electronics, owing to its large bandgap, smooth surface and stability. Polycrystalline hBN suffers from charge scattering at its grain boundaries, but single-crystal hBN avoids this problem and could be used to realize high-performance devices. However, the fabrication of large films is challenging, and an approach suitable for industrial

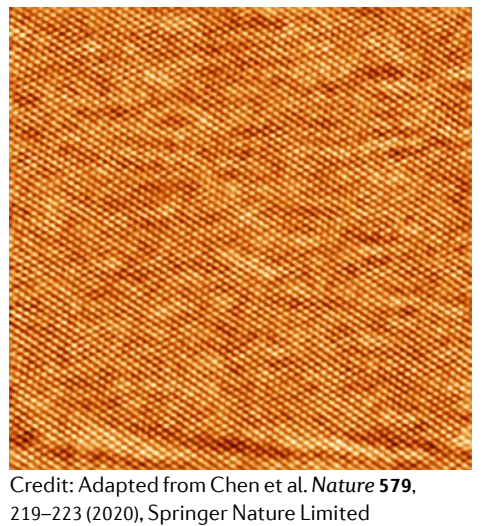

processing is needed. To date, a reliable approach to growing single-crystal hBN directly on wafers has not been demonstrated. Now, writing in Nature, Tse-An Chen and colleagues report the growth of single-crystal hBN monolayers on $\mathrm{Cu}(111)$ wafers.

Although hBN has previously been grown on $\mathrm{Cu}(111)$, if the $\mathrm{Cu}$ contains twin grains, the individual hBN flakes adopt two nearly degenerate configurations, resulting in hBN films with undesirable grain boundaries as the flakes merge. To avoid this issue, the team first prepared a single-crystal $\mathrm{Cu}(111)$ film devoid of twin grains on a 1-inch $c$-plane sapphire wafer. Subsequent high-temperature chemical vapour deposition of ammonia borane produces a mono-oriented hBN monolayer.

A clue to the success of the method comes from scanning tunnelling microscopy, which reveals numerous steps on the $\mathrm{Cu}(111)$ film.
First-principles calculations indicate that these steps affect the binding energy of hBN on the $\mathrm{Cu}(111)$ surface, leading Chen et al. to postulate that the energy difference between favourable and unfavourable configurations is large enough to ensure mono-oriented epitaxial growth.

The team demonstrated that their approach scales well by growing a single-crystal hBN monolayer on a 2-inch $\mathrm{Cu}(111)$ /sapphire substrate and successfully detaching the $\mathrm{hBN}$ layer from the substrate and transferring it to a 4-inch $\mathrm{SiO}_{2} / \mathrm{Si}$ wafer. Moreover, inserting a single-crystal hBN as an interface dielectric into a monolayer $\mathrm{MoS}_{2}$ field-effect transistor in a bottomgate configuration increased the electron mobility relative to devices containing either polycrystalline $\mathrm{hBN}$ or no hBN layer.

Claire Ashworth

ORIGINAL ARTICLE Chen, T.-A. et al. Wafer-scale single-crystal hexagonal boron nitride monolayers on $\mathrm{Cu}(111)$. Nature 579, 219-223 (2020) 